\title{
IMPLICATION ET SURIMPLICATION DU PRATICIEN-CHERCHEUR
}

\author{
Claire de Saint Martin ${ }^{\star}$ \\ Université de Cergy-Pontoise, Cergy-Pontoise, France
}

\section{RÉSUMÉ}

Selon l'analyse institutionnelle (Lourau), tout individu est impliqué par les institutions qu'il traverse et qui le traversent. Enseignante spécialisée exerçant dans une école élémentaire française, en classe spécialisée accueillant des enfants en situation de handicap mental, j'ai débuté une carrière de chercheuse à partir de mon questionnement quant aux violences qui s'exprimaient dans ma classe. Quand un praticien-chercheur entreprend une recherche sur son milieu professionnel, ses implication et surimplication opèrent un double mouvement, sur sa recherche et sur sa pratique. Ce mouvement a des effets sur ces deux milieux. Quels effets ont produit mon implication et ma surimplication dans ces deux champs professionnels?

Mots-clefs: analyse institutionnelle; implication; surimplication; praticienchercheur; violences scolaires.

\section{IMPLICATION AND OVER IMPLICATION OF THE PRACTITIONER-RESEARCHER}

\begin{abstract}
According to Institutional Analysis (Lourau), everyone is involved in the institutions through which he passes and that cross him. Special education teacher working in a French elementary school, in a class hosting children with mental disabilities, I began a career as a researcher from my questioning about the violences that were expressed in my class. When a practitionerresearcher undertakes a research on his workplace, its involvement and overinvolvement operate a double movement on his research and practice. This movement has effects on these two workplaces. What impact my involvement and my over-involvement in these professional fields have they produced?
\end{abstract}

Keywords: institutional analysis; involvement; over-involvement; practitionerresearcher; school violence.

\footnotetext{
‡ Doctorante 2ème année Science de l'Education. Adresse Postale : E.M.A. - Univ. de CergyPontoise/IUFM. ZAC des Barbanniers - Avenue Marcel Paul - F-92230 Gennevilliers. Mail: clairesm1709@hotmail.fr
} 
Je me propose ici de traiter de la question de l'implication du praticienchercheur et de ses effets, à partir d'une recherche que j'ai menée sur les violences dans une classe spécialisée accueillant des enfants en situation de handicap mental ou relevant de troubles envahissants du développement.

En France, le système scolaire français est divisé entre le premier degré (écoles primaire) et le second degré (collège, lycée). L'école primaire comprend l'école maternelle, non obligatoire, accueillant les élèves de trois à six ans et l'école élémentaire, accueillant les élèves de six à douze ans. Ces derniers sont répartis par âge dans cinq niveaux : le CP (Cours Préparatoire), le CE1 (Cours Elémentaire $1^{1 \text { ère } a n n e ́ e), ~ l e ~ C E 2 ~(c o u r s ~ e ́ l e ́ m e n t a i r e ~} 2^{\text {ème }}$ année), le CM1

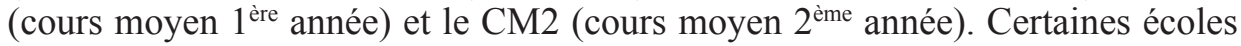
élémentaires accueillent aussi des élèves en situation de handicap dans des classes spécialisées, les CLIS, Classes pour L'Inclusion Scolaire. Il en existe quatre, selon le type de handicap :

CLIS 1 : classes destinées aux élèves dont la situation de handicap procède de troubles des fonctions cognitives ou mentales. En font partie les troubles envahissants du développement ainsi que les troubles spécifiques du langage et de la parole.

CLIS 2 : classes destinées aux élèves en situation de handicap auditif avec ou sans troubles associés.

CLIS 3 : classes destinées aux élèves en situation de handicap visuel avec ou sans troubles associés.

CLIS 4 : classes destinées aux élèves en situation de handicap moteur dont font partie les troubles dyspraxiques, avec ou sans troubles associés, ainsi qu'aux situations de plurihandicap (BULLETIN OFFICIEL n 31 du 27 août 2009).

Les CLIS accueillent un maximum de douze élèves (les classes ordinaires peuvent en compter de vingt à plus de trente), qui sont âgés entre six et douze ans. Les élèves qui y sont accueillis y restent plusieurs années et doivent connaître des temps d'inclusion en classe ordinaire, temps définis par l'enseignant en concertation avec celui de la classe ordinaire, selon le niveau scolaire de l'enfant et son aptitude à supporter le grand groupe. Un Assistant de Vie Scolaire (AVS), recruté sans formation, seconde l'enseignant dans la CLIS.

Je suis enseignante spécialisée auprès d'élèves dont la situation de handicap procède de troubles des fonctions cognitives ou mentales. Pour avoir le droit de travailler dans l'enseignement spécialisé, j'ai dû préparer un diplôme professionnel et, insatisfaite de la formation reçue, j'ai repris des études universitaires pour compléter celle-ci. J'ai enseigné six ans en CLIS 1 et la question des violences dans ma classe m'a conduite à étudier ce sujet de 
mémoire lors du master 2 que j'ai entrepris en 2010/2011. Ma démarche de recherche s'origine donc dans ma pratique. Je suis un exemple de praticienchercheur, doublement questionné par sa pratique et ses recherches.

Je traiterai dans une première partie de l'origine de mon questionnement, puis définirai les cadres théorique et méthodologique de ma recherche. La troisième partie s'intéressera à l'analyse des effets de ma double implication de praticien chercheur sur ma recherche.

\section{L'IMPLICATION DU PRATICIEN}

L'origine de mon questionnement vient plus particulièrement d'un élève, corrélé à la parution d'un rapport de l'INSERM ${ }^{1}$ paru en 2005.

\section{Le parcours de Mathieu}

Mathieu arrive en CLIS en septembre 2008, après trois ans de travail des équipes éducatives (en maternelle et au CP) pour convaincre ses parents de la validité de cette orientation. Ceux-ci considèrent cette classe comme un lieu transitoire de remise à niveau et envisagent la réintégration de leur fils en classe ordinaire dès son arrivée en CLIS. La maman surtout nie toute pathologie chez son fils, lui reconnaît des lenteurs d'apprentissage devant être comblées par son passage en classe spécialisée.

La CLIS compte 11 élèves de 7 à 12 ans : 1 fille et 10 garçons. Les débuts de Mathieu sont difficiles : il réclame constamment mon attention ou celle de l'AVS, ne peut agir en autonomie, ne soutient pas son attention, développe des relations principalement conflictuelles avec ses pairs.

La sollicitation de l'adulte se fait sur un mode envahissant : il demande à chaque instant s'il fait bien, s'il est sage, s'il est content de lui ; il intervient systématiquement quand je m'occupe d'un autre élève, ne peut faire aucune activité en autonomie. Quand il est placé dans cette situation, il interpelle un pair et le provoque, ou détourne la consigne et rend une production incohérente. Il ne supporte pas les activités groupales et le manifeste par un retrait visible (repli sur soi, regard dans le vide), des interventions sans rapport avec le sujet traité, des provocations à l'intention de ses pairs : il leur tire la langue, leur fait le «signe de mort " (index passé sur son cou, symbolisant un égorgement), les regarde en ricanant etc. Inclus en CP au cours de cette première année en numération/calcul et lecture, il progresse tout de même, grâce à un étayage individuel constant de l'enseignante. Il a aussi déjà fait un CP l'année précédente.

Il entre perpétuellement en conflit avec les autres élèves de la classe: petits coups portés chaque fois qu'il les croise, provocations verbales concernant essentiellement la déformation de leur prénom, moqueries systématiques en réaction à leurs paroles, rires intempestifs aux réflexions de l'enseignante à propos d'un autre élève. Dans la cour, tous les élèves se plaignent de son agressivité. Il se met en danger en provoquant physiquement et verbalement des enfants plus grands que lui. 
Chaque semaine, sa mère écrit dans le cahier de correspondance (cahier de liaison entre l'école et la famille) pour se plaindre du comportement des autres à l'égard de son fils, qu'elle affirme victime de harcèlements de la part plus particulièrement d'un élève désigné dans la classe, et de la part des autres élèves de l'école dans la cour et à la cantine. Ces harcèlements porteraient sur le fait que Mathieu est en CLIS : il serait en butte aux risées d'enfants qui le traiteraient régulièrement de "débile».

Dans la classe, le croisement du comportement de Mathieu et celui des autres génère des conflits permanents, m'obligeant à interrompre toute activité pour des rappels aux règles, décidées collectivement en début d'année, à imposer une discipline sévère, à limiter les temps collectifs. Ces conflits ne se traduisent pas par des violences extrêmes, mais par toute une série de paroles et gestes qui alourdissent le climat de la classe, ralentissent les apprentissages.

A la fin de cette première année, les parents acceptent le maintien en CLIS, sous réserve d'une inclusion plus grande en CE1.

La deuxième année ressemble à la première. Il y a toujours 11 élèves dans la classe, 1 fille et 10 garçons. 5 élèves sont partis (dont la fille) et 5 nouveaux sont arrivés, un de 6 ans et les autres de 7 ans. Mathieu a maintenant 9 ans et fait partie des plus âgés. Son comportement ne varie pas en CLIS. En inclusion, l'enseignant individualise les apprentissages de Mathieu et s'interroge : Mathieu ne semble pas stabiliser les acquis. Il réussit parfaitement une tâche un jour et perd tous ses moyens le lendemain sur la même tâche. En CLIS, il refuse ouvertement tout travail en autonomie, perturbe chaque activité, aidé par certains pairs qui lui répondent ou le provoquent volontiers. Il participe cependant plus aux activités, notamment lors du projet de classe : la réalisation d'un album musical. Sa mère continue à se plaindre régulièrement de l'agressivité des autres élèves à l'encontre de son fils. Elle réclame son retour en classe ordinaire pour l'année suivante, dénonce le handicap des autres élèves dans la faible progression de Mathieu qui «n'a visiblement pas le même problème qu'eux » et n'est pas porteur de handicap. Le comportement de Mathieu se dégrade au fil de l'année : provocations, coups portés aux autres, refus de travail. Au retour des vacances de Pâques, une réunion est organisée avec les parents, l'enseignant de CE1, le directeur et moi. L'enseignant de CE1 explique clairement l'impossibilité de retour en classe ordinaire, du fait des difficultés cognitives de Mathieu et j'insiste sur son comportement, notamment l'importance du regard d'autrui pour Mathieu, et son impossibilité d'agir hors de ce regard. A la suite de cette réunion, la mère de Mathieu écrit au directeur pour le remercier, remercier les enseignants pour leur implication et leur analyse. Elle décide de reprendre une thérapie pour Mathieu, abandonnée depuis deux ans.

En septembre 2010, je retrouve le même groupe que l'année précédente: un seul élève est parti, un autre est arrivé. Depuis la rentrée, Mathieu s'assagit considérablement. Il accepte les situations d'apprentissages, écoute plus et participe de façon cohérente et pertinente, s'implique dans le projet de classe (l'écriture d'un roman policier), peut agir en autonomie, provoque moins 
les autres. Maintenu dans la même classe en inclusion, il stabilise ses acquis et progresse plus régulièrement. Lors de l'équipe de suivi de scolarisation en novembre, la mère de Mathieu nous dit qu'elle est contente du chemin parcouru par son fils. Les mots dans le cahier de correspondance ont disparu.

La deuxième année de Mathieu en CLIS m'a amenée à m'interroger sur les situations conflictuelles permanentes au sein de la classe, que je pensais liées au fait que la CLIS accueillait alors des élèves diagnostiqués psychotiques et venant pour la majorité d'entre eux à temps partiel à l'école. L'arrivée de Mathieu, présentant des troubles des fonctions cognitives, m'ont conduite à m'intéresser aux ressorts de cette violence : elle n'était pas le seul fait des troubles envahissants du développement.

Les écrits récurrents de la mère de Mathieu m'ont interpellée, par la contradiction entre la réalité que vivait la classe au quotidien et les rapports que Mathieu en faisait à ses parents. Ange à la maison, victime des autres; démon en classe, provocateur de conflits. J'en ai d'abord conclu que Mathieu traduisait ainsi sa position impossible : sa mère niait son handicap et lui ne pouvait répondre à ses exigences. Sa violence ne pouvait qu'être l'expression d'une angoisse liée à cette contradiction. Mais il s'agit d'une interprétation confortable pour moi : du point de vue de l'enseignant, les difficultés des élèves trouvent souvent leur source dans l'environnement familial et social... D'autre part, l'agressivité des autres ne se justifie pas de la même façon. Serais-je alors à l'origine des conflits récurrents ? Comment réguler l'agressivité des élèves de ma classe ? Est-elle inhérente à la CLIS ? Aucune réponse d'ordre moral ou autoritaire n'apporte d'amélioration significative au climat de la classe. D'autre part, les violences exprimées ne sont pas spectaculaires et n'empêchent pas une socialisation et une solidarité au sein de la classe. Quelles sont alors la nature et l'enjeu de ces violences?

\section{Existe-t-il des pathologies violentes?}

Un rapport d'expertise collective de l'INSERM, «Troubles de conduite chez l'enfant et l'adolescent », est paru en septembre 2005, rédigé sur la base de plus de mille articles et documents. A partir de la notion de trouble de la conduite, introduite en 1968 dans le DSM-II (Diagnostic and Statistical Manual of Mental Disorder), ce rapport établit des relations entre ces troubles de la conduite et certaines pathologies.

L'une des pathologies psychiatriques les plus fréquemment associées à ce trouble est le trouble déficit de l'attention/ hyperactivité (TDAH). Certaines études épidémiologiques soulignent une continuité entre le TDAH de l'enfant et le trouble des conduites apparaissant à l'adolescence ; le TDAH durant l'enfance serait d'autant plus prédictif du trouble des conduites qu'il serait associé au trouble oppositionnel avec provocation (TOP) (INSERM, 2005, p. 7).

La violence enfantine relèverait donc directement de certaines pathologies. Le rapport relève aussi la corrélation entre trouble de la conduite et déficience intellectuelle. « Deux types de déficit neurocognitif semblent impliqués dans 
le trouble des conduites chez l'enfant et l'adolescent. Il s'agit, d'une part, d'un déficit des habiletés verbales et, d'autre part, d'un déficit du système d'inhibition exécutive de l'action » (INSERM, 2005, p. 23). Il différencie également la gravité comportementale en fonction de l'âge : les troubles de conduite précoces (avant 10 ans) se maintenant toute l'enfance et l'adolescence, -et du sexe, les garçons risquant de présenter des troubles de la personnalité à l'âge adulte. Ces troubles de conduite auraient un impact sur les violences scolaires. En s'appuyant sur ces études, le rapport de l'INSERM préconise finalement un dépistage précoce de ces troubles, de façon à prévenir la violence.

En novembre 2010, le rapport sur la prévention de la délinquance juvénile de Jean-Marie Bockel, secrétaire d'Etat à la justice, relève que:

Les études démontrent en effet que $15 \%$ des enfants sont vulnérables, c'est à dire incapables de répondre, de manière adaptée, à l'agressivité interne ou externe à laquelle ils sont exposés [...] Cette vulnérabilité pourrait pourtant être repérée chez les petits entre 2 et 3 ans. C'est à ce stade que doit être posé sur l'enfant un regard pluridisciplinaire visant à rechercher s'il existe à ces troubles une cause médicale ou familiale (BOCKEL, 2010, p. 43).

Le rapport ne précise pas sur quelles études il appuie sa démonstration. Il est à noter que c'est le ministère de la justice, et non celui de l'Education, qui a rédigé ce rapport.

L'expertise scientifique établissant un lien entre violence et pathologie est ainsi politiquement reprise dans une perspective préventive. Cependant l'individualisation ainsi faite des violences exprimées m'interrogeait, parce que mon expérience professionnelle me conduisait à envisager les facteurs environnementaux dans l'expression de ces violences. Je refusais aussi d'établir une causalité directe entre violence et pathologie. Je décidai alors d'entreprendre une recherche sur la question des violences en CLIS.

\section{CAdRe ThÉoriQue et MÉthodologie de la Recherche}

\section{L'analyse institutionnelle}

Ma recherche s'inscrit dans le cadre théorique de l'Analyse Institutionnelle, telle que la définit Lourau.

[...] l'analyse institutionnelle doit saisir l'action sociale dans son dynamisme et, sans préjuger du système institutionnel existant, chercher à dégager où est l'institution, c'est-àdire les rapports entre la rationalité établie (règles, formes sociales, codes) et les événements, développements, mouvements sociaux s'appuyant implicitement ou explicitement sur la rationalité établie et/ou la mettant en cause (LOURAU, 1970, p. 143). 
L'institution désigne donc une dynamique où interagissent tous les acteurs qui s'y trouvent, dans une interrelation de pouvoirs, d'intérêts multiples. «L'institution n'est pas «au-dessus» des pratiques, mais «à l'intérieur» » (MOURÃO et al., 2012, p. 135). Son analyse se fait par l'étude des implications, «c'est-à-dire des relations (libidinales, organisationnelles et idéologiques) qui s'établissent entre les sujets et les institutions. " (MONCEAU, 2012, p. 7). L'implication, en tant que rapport des sujets à l'institution est donc de fait et ne procède pas d'un choix. Dans le cadre d'une recherche menée par un praticien-chercheur sur son terrain professionnel, l'analyse de ses implications est particulièrement importante pour démêler " les positions enchevêtrées du praticien-qui-devient-chercheur " (KOHN, 2001, p. 15), ce qui était mon cas au commencement de cette recherche. Elle permet de « dénaturaliser et désindividualiser les analyses des relations quotidiennes, en nous détachant des opinions techniques pour nous orienter vers l'analyse des réseaux transversaux, des processus de subjectivation et des processus d'institutionnalisation » (NASCIMENTO; SCHEINVAR, 2012, p. 75).

Lourau distingue implication et surimplication. Cette dernière désigne un investissement conscient et affectif dans le travail. " La surimplication, elle, est l'idéologie normative du surtravail, de la nécessité de s'impliquer » (LOURAU, 1990, p. 113). De ce fait, elle masque souvent les implications, et traduit même un refus de les analyser. Cependant, dans le cadre de ma recherche, cette question de la surimplication a eu d'autant plus d'effets que ma double posture se marquait par un déséquilibre entre celle, experte, de praticienne et celle, novice, de chercheur. L'expression de praticien-chercheur "signifie que l'activité professionnelle génère et oriente l'activité de recherche, mais aussi de façon dialogique et récursive, que l'activité de recherche ressource et ré-oriente l'activité professionnelle » (DE LAVERGNE, 2007, p. 29). Pour réduire cette tension entre deux expériences, j'ai mené ma recherche dans une autre CLIS que celle où j'exerçais. Dans ma propre classe, il était impossible aux élèves de m'identifier à une posture de chercheur. De même comment observer des faits dans une situation où j'étais bien souvent surimpliquée?

\section{La méthodologie de la recherche}

Celle-ci s'est faite dans une grosse et vieille école de la région parisienne, qui regroupe dix-huit classes et la CLIS. Les 462 élèves sont réunis dans la même cour sur les temps de récréation. La CLIS est située au bout du couloir du premier étage de ce bâtiment tout en longueur, aux couloirs extrêmement sonores. Douze élèves de six à douze ans y sont accueillis, trois filles et neuf garçons. La majorité des enfants possèdent des troubles des fonctions cognitives, dont la plupart reste imprécise. Trois élèves ont été diagnostiqués comme relevant des troubles du comportement. Sur les douze élèves, seule une est incluse de façon conséquente en CE2. Deux élèves sont irrégulièrement inclus en sport dans des classes de CE2.

La méthodologie est donc une méthodologie qualitative et l'observation participante. Pendant treize demi-journées des mois de mars et avril $2011 \mathrm{~J}$ 'ai relevé et consigné scrupuleusement tous les faits délictueux que je voyais, en occupant le poste particulier et explicité de chercheur. «L'approche se veut 
holistique : l'observateur regarde et écoute, puis note les événements, les situant dans leur contexte ». (KOHN; NEGRE, 2003, p. 214) Il fallait cependant que ces observations n'interrompent pas l'action des protagonistes. "L'idée qu'en se faisant tout petit, au fond d'une classe, on évitera de modifier, par sa simple présence, la situation, est un mythe » (LAPASSADE, 1998, p. 57). Je me suis donc présentée aux élèves en précisant que j'étais moi-même enseignante en CLIS, que voir un autre fonctionnement m'intéressait, et en expliquant plus précisément mon projet : l'étude des disputes dans une classe. J'ai délibérément évité l'emploi du mot violence, pour ne pas les effrayer. Ensuite, en écrivant ouvertement et constamment, j'ai pu d'autant plus détourner l'attention des élèves, qu'ils ont vite compris que je ne les dénonçais jamais.

Après deux jours d'observation, je me suis plus particulièrement centrée sur quatre élèves qui présentaient à la fois suffisamment de diversités et de ressemblances pour permettre une analyse approfondie. Cette observation a été complétée par une réflexion collective avec les élèves sur la question de la violence. Les deux derniers jours, j'ai mené des entretiens individuels avec les enfants volontaires, sur la base de cinq questions : "As-tu des copains dans la classe? " "As-tu des copains dans l'école? " "Y a-t-il des élèves qui t'embêtent dans la classe? 》 "Y a-t-il des élèves qui sont embêtants dans la classe? 》 " Estce qu'il t'arrive parfois d'embêter les autres? »

Parallèlement, j'ai fait passer un questionnaire à tous les enseignants, pour connaître leurs représentations des élèves de CLIS, et j'ai mené des entretiens individuels enregistrés avec l'enseignante, la directrice et l'enseignant qui incluait l'élève de CLIS dans sa classe, et avait eu précédemment cette classe en charge.

Dans l'analyse institutionnelle, le journal est un outil central, un dispositif d'analyse. «La rédaction du journal potentialise et problématise les faits ignorés, considérés comme perturbateurs et déviants, considérés comme interdits ou bien de peu d'importance » (NASCIMENTO; SCHEINVAR, 2012 p. 78). Chaque soir, je transcrivais mes observations puis les analysais selon des points définis : les violences, ambiance et apprentissages, les adultes. La transcription me permettait de restituer le processus de chaque action notée et de mettre en lumière les enjeux et la réalité des interactions, mais aussi de mettre à jour mes propres implications et surimplications, déterminant les changements progressifs de mes observations.

\section{Analyse de mes implications et de SeS EFFets}

\section{Ma posture de praticien}

«L'observation ethnographique ne porte pas sur des univers d'individus mais sur des univers de relations. Travailler dans un milieu d'interconnaissance permet à l'enquête de ne pas piétiner, de ne pas avoir sans cesse à repartir de zéro » (BEAUD; WEBER, 2010[1997], p. 12). Le fait d'être enseignante de CLIS a facilité ma position de chercheur, en en justifiant l'objet de la recherche. Ma propre expérience légitimait mon questionnement aux yeux des acteurs de l'école observée. Ainsi, la directrice de l'école a accepté que je vienne dans l'école sans 
passer par l'obtention d'une autorisation de la hiérarchie, qui aurait nécessité des démarches lentes et compliquées. Connaissant l'institution Education nationale, je sais comment me présenter et agir auprès des enseignants et des élèves, parce que je possède les codes relationnels implicites qui régissent cette institution. Connaissant le fonctionnement d'une CLIS 1 , je savais quand et où porter mon attention pour observer les interactions sociales. Un avantage du chercheur praticien est de voir l'invisible. Cet avantage est renforcé lorsque le chercheur observe sur un autre lieu de travail que le sien. Ma position était évacuée des enjeux institutionnels de l'établissement, en même temps que ma connaissance du milieu me permettait la saisie et la compréhension de ces "phénomènes invisibles de l'extérieur » (MONCEAU, 1997, p. 97).

Il me fallait cependant éviter l'écueil des « allants de soi » professionnels pour que je puisse acquérir une véritable posture scientifique. Si ma fonction d'enseignante facilitait mon entrée dans l'école, il me fallait également me défaire des " automatismes " de ma profession. "Si le terrain est déjà connu du chercheur, nous entrons dans le domaine de la recherche impliquée, qui suppose une mise à distance, une démarche de défamiliarisation pour pouvoir voir autrement. » (KOHN; NEGRE, 2003, p. 91) Ayant une longue pratique du théâtre amateur, j'en ai utilisé les techniques de concentration me permettant cet équilibre entre distanciation et familiarisation. Le comédien est celui qui sait, mais qui sait aussi qu'il ne sait pas : une fois le texte appris, il doit l'oublier pour mieux le servir. Ici, en terrain familier, il me fallait rendre étrange le familier, pour éviter les préjugés, les interprétations hâtives suggérées par ma propre expérience professionnelle. Je m'y suis efforcée en maintenant mon attention sur les faits et gestes des élèves. En faisant le vide, je pouvais oublier les « allants de soi », porter un regard à la fois neuf et expérimenté sur les enfants. « Prenez la parole au ras du silence » écrivait Jacques Copeau (DASTE et al., 1999, p. 21). Cette technique me permettait une décentration de mon regard professionnel vers un regard scientifique et une centration exclusive sur le comportement et les paroles des élèves qui ont facilité « la mise en place d'une tension entre deux cadres de référence qui peut porter le processus de reconnaissance et de connaissance» (KOHN; NEGRE, 2003, p. 194).

\section{La question de la surimplication}

Dans le contexte spécifique de la CLIS 1, qui accueille des élèves fragiles, il n'était pas question de me positionner en chercheur extérieur, refusant tout contact. Cette posture aurait sensiblement modifié le comportement des élèves, de par son caractère possiblement anxiogène pour eux, la distance imposée alors pouvant être source d'inquiétudes et de retenue. De plus, le sujet même de la recherche m'obligeait à être acceptée par les élèves pour pouvoir en saisir les transgressions quotidiennes. En même temps, la venue d'un élément extérieur modifie nécessairement le quotidien de la classe. « Observateur et observé sont des êtres sociaux, ce qui pose le problème de leur positionnement réciproque en termes de distance sociale, à travers les rôles attribués dans la situation où ils sont engagés» (KOHN; NEGRE, 2003, p. 47). En utilisant mon savoir-faire 
professionnel, je me suis trouvée surimpliquée en tant que chercheuse. Pour permettre l'adaptation des élèves à ma présence, $\mathrm{j}$ ' ai progressivement participé aux activités de la classe en vérifiant d'abord le travail de certains élèves demandeurs, puis en soutenant individuellement les élèves qui en avaient besoin, et enfin en prenant en charge certaines activités les deux derniers jours. Cette participation active à la vie de la classe m'a rendue mobile et plus visible. De ce fait, les élèves ont cessé de s'intéresser à mes faits et gestes, qu'ils pouvaient contrôler à tout moment. Ici, ma visibilité m'a rendue d'autant plus « invisible » aux yeux des élèves, qu'ils se sont vite aperçus que la chercheuse ne les trahissait pas, ne dénonçant jamais leurs méfaits. La surimplication de la chercheuse a ainsi servi la recherche, en me permettant d'être le témoin privilégié de faits délictueux, les élèves cessant progressivement de tenir compte de ma présence.

Cependant, la surimplication de la praticienne a également eu des effets sur la recherche. Les entretiens formels que j'ai eus avec l'enseignante facilitaient le maintien de ma posture de chercheur par la présence du dictaphone et le fait que je parlais alors très peu, parce que ces entretiens étaient essentiellement descriptifs : le déroulement de la classe, le portrait des élèves, les violences dans la classe. Mais les nombreux entretiens informels, c'est-à-dire non explicitement reliés à ma recherche et non enregistrés ont mis à jour ma surimplication professionnelle par l'utilisation du regard du chercheur pour l'expertise du professionnel. En croisant mes propres observations et mon expérience professionnelle, $\mathrm{j}$ 'ai fait part de certaines de mes réflexions à Marie, l'enseignante, notamment en ce qui concerne la position de la directrice de l'école, le comportement d'un adulte à l'égard d'un élève, et du regard collectif porté sur ce même élève.

\section{Jeudi 3 mars 2011}

Marie [l'enseignante] explique la violence de Léo, par son âge mental de 2 ans (observation des psychologues) sur lequel elle insiste constamment, et son incapacité à se faire comprendre. Quand mes propres observations m 'amènent à lui faire remarquer que les compétences de Léo ne sont pas celles d'un enfant de deux ans (il s'exprime de façon intelligible, malgré des défauts d'élocution; il a du mal à comprendre les consignes, mais sait en suivre plusieurs quand il les a intégrées ; il a des compétences de niveau moyenne section en discrimination visuelle et en mathématiques), elle justifie ces progrès par son propre travail auprès de lui.

Le 25 mars, Marie m'envoie un mail dans lequel elle note que «Léo progresse à vue d'œil. » Ma surimplication professionnelle a donc eu des effets concrets sur la classe, puisqu'ici mes observations ont modifié le regard que Marie portait sur cet élève au vu d'une évaluation psychologique.

\section{Les effets sur le chercheur}

J'ai écrit mon journal de recherche en deux temps. J'ai d'abord noté tout ce que je voyais, en m'efforçant de porter un regard neuf, sans sélection des violences. Celle-ci s'est faite plus tard, au moment de l'analyse, dans lesquelles je distinguais aussi mes émotions et sensations, mes implications. 
L'observateur entend restituer la situation qu'il a pu connaître telle quelle, en la suivant plutôt qu'en la cadrant, en en rendant compte par transposition plutôt que par décomposition et reconstitution, en ajustant ses coordonnées par recours à un autre angle de vue plutôt qu'en s'efforçant d'en fixer les mesures par rapport à un étalon uniforme et extérieur (KOHN; NEGRE, 2003, p. 196).

Cette volonté de ne pas trier en situation m'a permis de dégager des causes que je ne soupçonnais pas, d'identifier et de caractériser certains faits. Ainsi, la majorité des faits caractérisés comme violents correspondent plus souvent à ce que j'ai nommé des manifestations d'hostilité et des diversions transgressives.

Le choix même des observations faites dépendait de mes implications et surimplications. Ainsi, au début de la recherche, le comportement de l'intervenante sportive même m'a interpellée, me renvoyant à la surimplication de l'enseignante.

Lundi 28 février 2011

La séance de sport a été éprouvante pour moi : l'intervenante s'est focalisée sur Léo, qui suivait peu le groupe, toujours en termes dépréciatifs "il ne peut pas ; comment lui faire suivre le cross? " "C'est pas gagné avec lui 》, "Cours Forrest »; toutes ses actions étaient commentées à l'adresse de Marie. Un prof se met à la fenêtre du premier étage et l'intervenante sportive l'interpelle "Regarde Léo, comme il court bien! ». Ironie constante, que Marie écoute sans riposter ; elle aide une fois Léo à faire le tour de la cour. Ces commentaires étaient faits, sans tenir compte des élèves autour d'elle. Grrrr.

L'observation suppose plusieurs actes qui ne doivent pas être pensés selon un ordonnancement purement linéaire, mais bien en interactions constantes. Aussi bien, nous ne marquons pas de démarcation nette avec la situation dans laquelle plonge l'observation, et la démarche de représentation qui la prolonge ou la structure (KOHN; NEGRE, 2003, p. 42).

Mon indignation devant le comportement de l'intervenante sportive a nui d'une certaine façon à mes observations suivantes. Je notais dans mon journal :

\section{Lundi 7 mars 2011}

J'ai très peu noté les faits des élèves durant la séance de sport, tellement l'intervenante sportive m'énervait. Je me rends compte que, prise par mon jugement sur elle, je n'ai pas pu tenir ma position de chercheur à ce moment-là, parce que je n'ai fait que guetter ses interventions auprès de Léo, qu'elle affuble du surnom ridicule de "Lolo».

Même dans le temps de l'écriture apparaît ici ma surimplication. Mais, en distinguant ainsi mes implications et surimplications, au regard de cet analyseur qu'était le comportement de cet adulte, j'ai également pu mettre à jour la responsabilité institutionnelle des violences en CLIS. 
Cette responsabilité institutionnelle des violences, responsabilités au sein de la classe (autorité de l'enseignant), de l'école (équipe pédagogique, directeur), de l'Etat (directives ministérielles), définit les violences scolaires comme un fait social, une production de l'institution. En même temps, l'absence de violences spectaculaires dans la classe observée, la multiplication de faits délictueux mineurs m'ont permis d'en comprendre leurs enjeux en termes de socialisation.

La violence à l'école élémentaire se définit déjà dans le rapport aux pairs. Simultanément, elle montre l'importance des relations entre pairs pour les écoliers, relations le plus souvent clandestines au sein de la classe mais trouvant à s'exprimer dans la cour de récréation (CARRA, 2009, p. 54).

Au terme de ma recherche, je pouvais définitivement remettre en cause une relation exclusive entre violence et pathologie.

\section{Conclusion}

Cette recherche a eu un double effet sur ma posture de praticien-chercheur, dans un mouvement circulaire. Initiée à partir de ma propre pratique, elle m'a permis de construire une identité de chercheur. Mais elle a aussi eu des effets sur ma propre pratique. De retour dans ma propre classe, j'ai pu constater une modification de l'ambiance de la classe par le simple changement de ma posture professionnelle, lié aux découvertes de ma recherche, sans que j'aie eu à mettre en œuvre des actions spécifiques.

La double implication et surimplication du praticien-chercheur dans ce mouvement produit donc des effets scientifiques, par la mise à jour, la caractérisation et la critique des faits dits « violents » dans la CLIS. Mais elle a également une portée pédagogique puisqu'en prenant conscience des responsabilités institutionnelles dans l'expression de ces faits, j'ai pu apaiser l'atmosphère de ma classe et changer sensiblement les relations entre tous ces acteurs.

\section{Note}

${ }^{1}$ L'INSERM, Institut National de la Santé et la Recherche Médicale, est un organisme de recherche public placé sous la double tutelle du ministère de la recherche et de celui de la santé. Il a pour objet l'étude de la santé humaine et l'expertise constitue une de ses missions. 


\section{RÉFÉRENCES}

BEAUD, S.; WEBER, F. Guide de l'enquête de terrain (1997). Paris : La Découverte, 2010.

BOCKEL, J. M. (Secrétaire d'Etat à la Justice). La prévention de la délinquance des jeunes. Rapport à Monsieur le Président de la République, nov. 2010.

BULLETIN OFFICIEL $\mathrm{n}^{\circ} 31$ du 27 août 2009. Scolarisation des élèves handicapés à l'école primaire; actualisation de l'organisation des classes pour l'inclusion scolaire (CLIS). Circulaire $\mathrm{n}^{\circ} 2009-087 \mathrm{du} 17-$ 7-2009. Disponible à l'adresse: <http://www.education.gouv.fr/cid42618/ mene0915406c.html>. Consulté le 21 sept. 2013.

CARRA, C. Violences à l'école élémentaire. Paris : PUF, 2009.

DASTE, C. (Dir.) et al. Anthologie subjective Jacques Copeau. Paris: Gallimard, 1999.

DE LAVERGNE, C. La posture du praticien-chercheur : un analyseur de l'évolution de la recherche qualitative. Recherches Qualitatives - Hors Sérien. 3, p. 28-43, 2007. Actes du colloque BILAN ET PROSPECTIVES DE LA RECHERCHE QUALITATITVE,

INSERM. Troubles des conduites chez l'enfant et l'adolescent, Expertise collective Synthèse. Paris: Les éditions Inserm, 2005.

KOHN, R. C. Les positions enchevêtrées du praticien-qui-devient-chercheur. In: MACKIEWICZ, M. P. (Coord.). Praticien et chercheur: parcours dans le champ social. Paris: L'Harmattan, 2001. p. 15-38.

KOHN, R. C.; NEGRE, P. Les Voies de l'observation. Paris : L'Harmattan, 2003.

LAPASSADE, G. Microsociologie de la vie scolaire. Paris : Economica, 1998.

LOURAU, R. L'analyse institutionnelle. Paris: Minuit, 1970.

LOURAU, R. Implication et surimplication. La revue du Mauss, Paris, n. 10, p. 110-119, 1990.

MONCEAU, G. Le concept de résistance en éducation. Paris : ANRT, 1997.

MONCEAU, G. (Dir.). L'analyse institutionnelle des pratiques: une socioclinique des tourments institutionnels au Brésil et en France. Paris: L'Harmattan, 2012. 
MOURÃO, L. C. et al. Analyser l'implication des professionnels en Santé collective en accompagnant les équipes. Comment la découverte des implications transforme la pratique collective ? In : MONCEAU, G. (Dir.). L'analyse institutionnelle des pratiques: une socio-clinique des tourments institutionnels au Brésil et en France. Paris: L'Harmattan, 2012. p. 133-149.

NASCIMENTO, M. L.; SCHEINVAR, E. Formation professionnelle et outils de l'analyse institutionnelle en formation de psychologues. In: MONCEAU, G. (Dir.). L'analyse institutionnelle des pratiques: une socio-clinique des tourments institutionnels au Brésil et en France. Paris : L'Harmattan, 2012. p. 71-81.

Reçu: Septembre 30, 2013 Accepté: Décembre 29, 2013 